\title{
LA ATENCIÓN PRENATAL: UNA MIRADA DESDE LOS PROFESIONALES DE LA SALUD. ESTUDIO CUALITATIVO. BUCARAMANGA (COLOMBIA), 2012
}

\section{Pre-natal care seen from the perspective of healthcare practitioners. Bucaramanga, Colombia, 2012}

Flor de María Cáceres-Manrique, $\mathrm{PhD}^{1}$; Myriam Ruiz-Rodríguez, $\mathrm{PhD}{ }^{2}$; Andrea Hernández-Quirama, $\mathbf{P h D}{ }^{3}$

Recibido: octubre 13/14 - Aceptado: mayo 28/15

\section{RESUMEN}

Objetivo: comprender los significados que tiene la atención prenatal (AP) para los profesionales de la salud (PS) que atienden a las embarazadas en control prenatal.

Materiales y métodos: investigación cualitativa, con sustento teórico en interaccionismo simbólico y métodos de la teoría fundamentada (TF). Previo consentimiento informado se realizaron entrevistas en profundidad a 17 PS: médicos, ginecólogos, perinatólogos, enfermeras y psicólogas, quienes realizaban AP en instituciones públicas (centros de salud de atención primaria) y privadas (centros médicos y consultorios) de Bucaramanga (Colombia), en 2012. En estos escenarios también se observaron las interacciones de los profesionales y las gestantes. La información fue analizada simultáneamente con su

1 Enfermera; doctora en Salud Pública; profesora asociada, Escuela de Medicina, Departamento de Salud Pública, Universidad Industrial de Santander, Bucaramanga (Colombia). Grupo de investigación GUINDESS, Departamento de Salud Pública, Escuela de Medicina, Universidad Industrial de Santander, Bucaramanga (Colombia). fmacer@uis.edu.co

2 Enfermera; doctora en Salud Pública con énfasis en sistemas de salud; profesora titular, Escuela de Medicina, Departamento de Salud Pública, Universidad Industrial de Santander, Bucaramanga (Colombia).

3 Trabajadora social; doctora en estudios feministas y género, profesora asistente, Escuela de Trabajo Social, Universidad Industrial de Santander, Bucaramanga (Colombia). recolección, usando razonamiento lógico inductivo y métodos propios de la TF para generar categorías, y muestreo teórico hasta lograr saturación de las categorías emergentes. El estudio fue aprobado por los comités de ética de las instituciones participantes. Resultados: emergieron tres categorías denominadas: 1) atención centrada en el manejo del riesgo, 2) dualidad entre las fallas del sistema y la calidad de la atención y 3) atención prenatal como un espacio de interacción y satisfacción.

Conclusiones: los participantes perciben la AP en tres sentidos: como escenario técnico, como oportunidad de interacción y satisfacción, y como una preocupación por lograr franquear las barreras que impone el sistema y brindar atención de buena calidad a las gestantes.

Palabras clave: atención prenatal, gestión de riesgos, personal de salud, satisfacción personal.

\section{ABSTRACT}

Objective: To understand the meaning of prenatal care (PC) for the different healthcare professionals (HCP) who see pregnant women in antenatal consultation.

Materials and methods: Qualitative research supported theoretically on Symbolic Interactionism and Grounded Theory (GT). After informed 
consents were obtained, in-depth interviews were conducted among 15 HCPs including physicians, gynaecologists, perinatologists, nurses and psychologists providing $\mathrm{PC}$ in public (primary care clinics) and private (medical centres and offices) institutions in Bucaramanga, Colombia, in 2012. Interactions between professionals and pregnant women were also observed in those settings. The information was analysed concurrently as it was gathered using inductive logic reasoning and GT methods in order to generate categories; logic sampling was then applied until saturation of the emerging categories was achieved. The study was approved by the Ethics Committees of the participating institutions.

Results: Three categories emerged: 1) Risk management-focused care; 2) Dichotomy between the failings of the system and care quality; and 3) Prenatal care as a setting for interaction and satisfaction. Conclusions: The participants award meaning to PC from three perspectives: as a technical setting, as an opportunity for interaction and satisfaction, and as a motivation to overcome the barriers imposed by the system and provide good-quality care during pregnancy.

Key words: Pre-natal care, risk management, healthcare staff, personal satisfaction.

\section{INTRODUCCIÓN}

La atención prenatal (AP) es catalogada por la Organización Mundial de la Salud (OMS) como una estrategia prioritaria en salud pública, pues se lleva a cabo con el fin de brindar atención integral a la mujer gestante y a su hijo por nacer (1). En este sentido, ha sido valorada como una estrategia exitosa, siempre y cuando se garantice una intervención integral, que incluya atención con calidad, educación continuada y atención especializada desde el primer nivel (2). Para la realización de la AP se han establecido guías de atención (3), en las cuales se proveen herramientas para evaluar el riesgo biopsicosocial de las gestantes y se considera importante la interacción entre ellas y los profesionales de la salud (PS) que las atienden. Esta interacción está permeada por múltiples facto- res, entre ellos los culturales, sociales y económicos $(4,5)$, y por los significados que orientan las prácticas y formas de actuar de los PS.

De acuerdo con los planteamientos de Blumer (6), los significados se construyen en la interacción con las demás personas y están influenciados por las creencias, los valores y el contexto en el cual se desenvuelven. De esta manera, el significado se convierte en la fuerza que orienta a las personas para actuar en los diferentes escenarios y desarrollar distintos roles (6). Visto así, los servicios de salud son una construcción social, que se produce en una trama compleja de significados y relaciones entre los diversos actores; por ello, es relevante conocer los significados que mueven a los actores sociales, quienes desde sus marcos de sentido construyen y observan la realidad (7).

En el ámbito de la investigación sobre AP, ha habido un predominio de los estudios que se enfocan a la evaluación de los servicios (7) desde la perspectiva de las usuarias (8), pero se han dejado de lado los pensamientos y sentimientos de los PS de la salud que las atienden. Esta información ofrece a los PS de ginecología y obstetricia, y a los demás profesionales de la salud involucrados en la AP, una oportunidad para reflexionar sobre lo que significa su quehacer y tomar conciencia de que sus percepciones y prácticas van redefiniendo y reconstruyendo la AP en el día a día. También pone de manifiesto a los tomadores de decisiones cómo, desde la cotidianidad de la AP, se pueden identificar las distintas dimensiones que están presentes en la atención y la necesidad de rescatar la dimensión subjetiva de los PS para realizar ajustes a la política de AP.

Por tanto, el objetivo de este estudio fue comprender los significados que un grupo de profesionales de la salud le dan a la atención primaria en Bucaramanga (Colombia).

\section{MATERIALES Y MÉTODOS}

Investigación cualitativa, con sustento teórico en el interaccionismo simbólico (6) y el uso de los 
métodos de la teoría fundamentada en los datos (9-11). Previo consentimiento informado, se entrevistaron profesionales de diferentes disciplinas del área de la salud, quienes se desempeñaban en AP a gestantes en instituciones públicas (centros de salud de atención primaria) y privadas (centros médicos y consultorios) en la ciudad de Bucaramanga, durante el año 2012. Los participantes fueron seleccionados de forma intencional para que sirvieran de informantes clave, buscando la pluralidad de actores que pudieran dar cuenta de diferentes percepciones sobre la AP desde diversos escenarios. Fueron contactados en los sitios donde llevaban a cabo la AP.

Para la realización de las entrevistas se diseñó una guía con preguntas abiertas y amplias que permitieran a los participantes expresarse libremente. Las entrevistas fueron llevadas a cabo en los lugares escogidos por los participantes, y grabadas previa autorización del entrevistado, tuvieron una duración de 90 minutos en promedio y, en doce casos, se requirió más de un encuentro con la persona entrevistada para completar la información. También se llevó a cabo observación participante y no participante, de las interacciones entre los PS y las embarazadas en los consultorios y en los sitios de atención, la cual fue registrada en los diarios de campo.

Tanto las entrevistas como los diarios de campo, fueron transcritas en archivos de texto, editadas y analizadas. Para ello, los textos fueron leídos, línea por línea, por dos de las investigadoras, quienes generaron códigos, usando un sistema de codificación manual abierta y de comparación constante bajo el razonamiento lógico inductivo propio de la TF, para generar categorías, seguido del muestreo teórico, hasta lograr saturación de las categorías emergentes, de acuerdo con las recomendaciones para el manejo de datos cualitativos (9-11). Los resultados se validaron en grupos focales de PS de la ciudad donde se llevó a cabo el estudio.

La investigación fue aprobada por los Comités de Ética de las instituciones participantes.

\section{RESULTADOS}

Se entrevistaron 17 profesionales, once mujeres y seis hombres: cinco enfermeras, cuatro médicos generales, cuatro ginecólogos, dos perinatólogos y dos psicólogas. Las edades de los participantes estaban entre 26 y 61 años, con un promedio de 42 años y una mediana de 45. Al momento de la entrevista, todos los hombres y siete de las once mujeres tenían hijos.

De sus discursos y de las observaciones realizadas, emergieron tres categorías: 1) Atención centrada en el manejo del riesgo, 2) Dualidad entre las fallas del sistema y la calidad de la atención, 3) Atención prenatal como espacio de interacción y satisfacción. A continuación se analiza cada una de ellas.

1. Atención centrada en el manejo del riesgo. Para 16 de los 17 profesionales, la atención a la gestante está centrada en la detección temprana de los riesgos porque, aunque se considera baja la posibilidad de que un embarazo tenga complicaciones, ellos tienen claro que existen riesgos que pueden ser detectados e intervenidos en forma oportuna para evitar que se desarrollen patologías o complicaciones que puedan afectar la salud de la mamá o del bebé. Esto se confirmó en la observación, donde la preocupación central del profesional estaba dirigida a la búsqueda de signos y síntomas de la gestante, que le indicaran que había un riesgo. Además, se expresó en frases como: "La atención prenatal comprende una serie de actividades encaminadas a detectar los riesgos en forma precoz y a suministrar tratamiento oportuno, para prevenir complicaciones de la mujer o de su hijo" (enfermera, sector público).

Dado que la AP está centrada en la búsqueda de riesgos, el objetivo principal de los PS, desde su primer contacto con las gestantes, está orientado a clasificarlas y a actuar en consecuencia, tal como ellos lo relatan: "Uno como médico debe clasificarlas según el riesgo y actuar de acuerdo al mismo: enviarlas al especialista y hacerles seguimiento" (médica general, sector público).

El énfasis en los riesgos lleva a que los PS concentren sus esfuerzos en que las gestantes aprendan a identificarlos. Esto se observó en los escenarios de 
atención, donde los PS realizan estrategias educativas con énfasis en la enseñanza del reconocimiento de los signos de alarma por parte de la gestante, le entregan folletos y son reiterativos en el tema. Al respecto, una enfermera y una psicóloga expresaron: "Estamos dados a la tarea de educarlas para que logren tener una buena salud y disminuir los riesgos de muerte o de enfermedades que pueden ser prevenibles si se detectan a tiempo" (enfermera, sector público). "Lo que buscamos es prevenir cualquier enfermedad del bebé o de la madre. Tenemos un equipo interdisciplinario bien preparado, para educarlas" (psicóloga, sector privado).

La preeminencia sobre lo biológico deja en un segundo plano otro tipo de riesgos, principalmente por parte de los profesionales de medicina. Esto se confirma en los siguientes relatos: "Uno se dedica a la parte clínica y sobre todo a determinar factores de riesgo. La parte psicosocial le corresponde a otro profesional" (médico general, sector público).

"A las embarazadas las acompaño en un proceso biológico que es tal vez el más importante por el cual atraviesa la vida de la mujer" (ginecóloga, sector privado).

Con el ejercicio profesional basado en identificar y manejar los riesgos, los PS consideran que cumplen su meta: "Al identificar y controlar los riesgos uno asegura un buen resultado: un parto con un bebé sano, una madre sana, que es lo que uno quiere" (ginecólogo, sector privado).

2. Dualidad entre las fallas del sistema y la calidad de la atención. Una segunda categoría de significados fue expresada por 16 de los 17 participantes, quienes reconocieron que en algunos escenarios hay dificultades para la prestación de la AP con calidad, como el exceso de trámites y requisitos administrativos que las empresas promotoras de salud (EPS) imponen para atender a las gestantes. Da cuenta de lo anterior el siguiente testimonio: "Hay dificultades porque ellas tienen que ir de un lado a otro, a pedir autorizaciones, pagar cuotas, sacar citas, tomarse exámenes y reclamar resultados" (enfermera, sector público).
Los PS que viven esos obstáculos sienten frustración, dado que ello limita su acción como garantes de una buena atención, así lo relatan: "Algunas EPS contratan los exámenes con una IPS, la consulta con otra, las ecografías con otra, y ponen a las gestantes a dar vueltas. Eso las desmotiva a ellas, y a nosotros nos frustra" (médico general, sector público).

Asimismo, los PS reconocen que hay otras barreras de diferente índole que complejizan la prestación de una AP plena, satisfactoria y con calidad. Así lo relatan: "Hay algunas pacientes que tienen poca fe en la medicina occidental, poca credibilidad, o que han tenido experiencias negativas, y no les gusta ir a consulta" (perinatólogo, sector privado).

Ante los obstáculos mencionados, los PS experimentan la necesidad imperiosa de aportar su mejor esfuerzo para ofrecer AP con calidad, como una forma de compensar las limitaciones propias del sistema. Así lo refieren: "Para nosotros lo primordial es dar una buena atención. Por eso les ayudamos con el papeleo, los trámites, las citas, y si no tienen carné las atendemos y las orientamos para conseguir la afiliación" (médico general, sector público).

De otro lado, independientemente de la profesión y del nivel académico, los participantes consideran que la presencia de barreras en el sistema de salud es diferencial según el tipo de afiliación de la gestante y tiene consecuencias graves. Así lo relatan: "Hay tantas barreras y trámites que terminan afectando la salud de las señoras. Son tantas y tan complejas, que cuando ellas consiguen atención, ya la complicación se ha dado, cuando se hubiera podido prevenir" (ginecóloga, sector público).

3. Atención prenatal como espacio de interacción y satisfacción. La tercera categoría, manifestada por 14 de los 17 participantes, es la AP como satisfacción, dado que se configura como un escenario agradable de interacción, realización profesional y crecimiento mutuo, mediado por una comunicación asertiva. Este significado lleva al PS a asumir el reto de brindar seguridad a sus pacientes, y evitarles el estrés y las complicaciones. Esta situación se evidenció en la interacción con las gestantes, donde los PS se 
esfuerzan por ser cálidos, amables y claros en las recomendaciones que les brindan. Así lo refieren: "[La AP] es un espacio de encuentro con las embarazadas, es un lugar de crecimiento mutuo, tanto de nosotros como de ellas" (enfermera, sector público). Un especialista manifiesta: "Es una ocasión de encuentro muy agradable y uno empieza a tener una comunicación muy asertiva con ellas" (ginecólogo, sector privado).

En general, los PS manifiestan un gusto especial por el ejercicio de la AP y por la gratitud de las gestantes. Así lo expresan: "Del CPN [control prenatal] destaco la oportunidad de interactuar con las mujeres y la gratitud de ellas por las enseñanzas recibidas, independiente del resultado del embarazo" (enfermero, sector público). "Cuando ya el bebé nace y ellas vienen a vacunarlo o a control, [...] uno ve en su cara el afecto, el agradecimiento y la satisfacción” (enfermera, sector público).

\section{DISCUSIÓN}

Para los participantes en este estudio, la AP tiene tres significados: como un escenario eminentemente biomédico con enfoque en prevención, como una oportunidad de realización y satisfacción personal, y como un espacio en el cual ellos deben hacer un esfuerzo para tratar de equilibrar, con buena atención, las distintas dificultades que el sistema impone a las gestantes.

La AP, como escenario biomédico, representa un reto para los PS en cuanto a los resultados de salud del binomio madre-hijo. En ese sentido, se pudo constatar el alto significado que ellos dan a la identificación temprana de riesgos y a la preocupación constante por garantizar un buen estado de salud. Este resultado es consistente con hallazgos de otros autores $(5,12)$, lo cual sugiere que el enfoque biomédico, o enfoque de riesgos, predomina en la práctica; no obstante, existen otras miradas, como aquellas centradas en los determinantes sociales (13) y nuevas reflexiones que invitan a explorar no solo el componente biológico, sino también las condiciones socioculturales de las personas para obtener resultados más sostenibles y equitativos en salud (14). Este significado de la AP centrada en la prevención de los riesgos podría estar relacionado con la educación recibida y el énfasis que el sistema de salud hace en la evaluación de los profesionales sobre la detección de riesgos, especialmente biológicos, dejando en segundo plano los riesgos psicológicos, familiares, sociales y contextuales que también influyen, tanto en los resultados de salud madre-hijo, como en la calidad de interacción entre los profesionales y la gestante.

De otro lado, los participantes percibieron la AP como un escenario de realización y satisfacción, a pesar de las limitaciones y los obstáculos para la prestación del servicio con calidad. Resultados similares se han encontrado en estudios previos como el de Rhamani y Brekke (4), cuyos hallazgos dan cuenta que los PS, a pesar de las difíciles condiciones laborales, se sienten orgullosos de su trabajo. En esta investigación, la realización y satisfacción manifestada por los PS es el resultado del balance positivo que hacen entre la oportunidad de interactuar con las gestantes, la eficacia de las enseñanzas, la consejería que les brindan, el reconocimiento que las gestantes hacen de la atención brindada y la mitigación de las limitaciones y barreras que el sistema plantea a sus usuarios, tal como ha sido reportado en estudios previos (15). Otras investigaciones han encontrado resultados similares, tanto en Colombia como en otros países (16-17), donde se han puesto de manifiesto los dilemas y las tensiones que enfrentan los PS al momento de querer brindar una AP con calidad, en un sistema cuya estructura está orientada al mercado y a la obtención de lucro, más que a la solución de las necesidades (18), en este caso, de las gestantes.

El hallazgo sobre la percepción del control prenatal como un escenario de interacción es consistente con la perspectiva de las usuarias de la AP abordada en otros estudios, como el de Jallow et al. (8). Dicho estudio, y los resultados presentados en este artículo, sugieren la importancia de fomentar y desarrollar habilidades comunicativas en los pro- 
gramas de formación de pregrado y posgrado y en los mismos servicios de salud (14). La calidad de la interrelación personal y de la información que se comparte durante los encuentros entre PS y gestantes depende tanto de su formación y compromiso como de una serie de decisiones gerenciales como la supervisión, la descripción del trabajo, el cumplimiento de las guías y, sobre todo, del entrenamiento en herramientas y habilidades comunicativas (19).

En este sentido, políticas de formación en comunicación asertiva y políticas institucionales para promover este tipo de comunicación propiciarían una mirada más integradora a la salud de la gestante y su hijo, no solo desde lo biológico, sino desde lo psicosocial, con resultados más productivos tanto para los PS como para las gestantes, sus familias y la sociedad, tal como lo han señalado varios autores en estudios previos (20-22).

Las fortalezas de este estudio están dadas por la participación de profesionales de las diversas disciplinas y grados de formación, de diferentes niveles de atención, tanto públicos como privados, todos ellos involucrados en la AP a gestantes de distintos estratos socioeconómicos de la ciudad, además de la triangulación de fuentes y métodos de investigación. En contraste, las debilidades están referidas a las características implícitas del tipo de estudio, pues dado que está basado en la subjetividad de los participantes, sus resultados no pueden ser generalizados y extrapolables a otros contextos, sin el examen consciente de las implicaciones epistemológicas propias del método per se. La invitación, en este sentido, es a considerar que esta es una interpretación del asunto ajustada al contexto, que de acuerdo con el interaccionismo simbólico (5) es dinámico y sensible a cambios y a nuevas condiciones de los actores, de ahí la necesidad de hacer otras aproximaciones en distintos escenarios para contrastar los resultados.

\section{CONCLUSIÓN}

Los hallazgos de este estudio dan cuenta de los distintos significados que los participantes dan a la
AP: como una oportunidad de interacción y satisfacción; como un escenario técnico, y, finalmente, como una preocupación constante por lograr el balance entre las barreras que la funcionalidad del sistema de salud colombiano impone a las gestantes y la necesidad de ofrecer una atención prenatal de buena calidad.

\section{AGRADECIMIENTOS}

Las autoras agradecen a los profesionales de la salud, quienes aceptaron participar tanto en las entrevistas como en la validación de resultados. A la Universidad Industrial de Santander y a la Universidad de Antioquia por el apoyo en el desarrollo de la investigación.

Nota: estos resultados forman parte de la tesis doctoral de Flor de María Cáceres-Manrique, cofinanciada por el Departamento de Salud Pública de la Universidad Industrial de Santander y el Fondo Profesoral de la Universidad de Antioquia.

\section{REFERENCIAS}

1. Organización Mundial de la Salud (OMS). Informe sobre la salud en el mundo 2005 - iCada madre y cada niño contarán! Ginebra: OMS; 2005.

2. Pazmiño S, Guzmán-Gómez N. Control prenatal y su impacto en la mortalidad materna. Análisis de una tendencia, 1994-2004. Cali, Colombia. Rev Colomb Obstet Ginecol. 2009;60:12-8

3. Ministerio de Salud y de Protección Social-Colciencias. Guías de Práctica Clínica para la prevención, detección temprana y tratamiento de las complicaciones del embarazo, parto o puerperio. Guías No. 11-15. Colombia 2013 [visitado 2015 May 15]. Disponible en: http://gpc.minsalud.gov.co/Documents/Guias-PDFRecursos/Embarazo/GPC_Comple_Embarazo.pdf

4. Rahmani Z, Brekke M. Antenatal and obstetric care in Afghanistan - a qualitative study among health care receivers and health care providers. BMC Health Services Research 2013 [visitado 2014 Sept 20]; 13:166. Disponible en: http://www.biomedcentral. com/1472-6963/13/166 
5. Lasso-Toro P. Atención prenatal: ¿tensiones o rutas de posibilidad entre la cultura y el sistema de salud? Pensamiento Psicológico. 2012;10:123-33.

6. Blumer H. El interaccionismo simbólico: perspectiva y método. Barcelona: Hora SA; 1969.

7. Delgado-Gallego ME, Vázquez-Navarrete ML, De Moraes-Vanderlei L. Calidad en los servicios de salud desde los marcos de sentido de diferentes actores sociales en Colombia y Brasil. Rev Salud Pública. 2010; 12:533-45.

8. Jallow IK, Chou YJ, Liu TL, Huang N. Women's perception of antenatal care services in public and private clinics in the Gambia. Int J Qual Health Care. 2012;24:595-60;24:595-600.

9. Strauss A, Corbin J. Bases de la Investigación Cualitativa. Técnicas y procedimientos para desarrollar la teoría fundamentada. Medellín: Universidad de Antioquia; 2002.

10. Corbin JM, Strauss AL. Basics of qualitative research: Techniques and procedures for developing grounded theory. Thousand Oaks: Sage Publications; 2008.

11. Charmaz K. Constructing grounded theory. A practical guide through qualitative analysis. 2 ed. London: Sage Publications; 2014.

12. Argote LA, Velásquez ML. Ante la desesperanza del desplazamiento: un hijo sano, el mayor anhelo de la mujer gestante. Colomb Méd. 2007;38:S27-S43.

13. Organización Mundial de la Salud. Comisión sobre Determinantes Sociales de la Salud - Informe Final. Subsanar las desigualdades en una generación [visitado 2015 May 15]. Disponible en: http://www.who.int/ social_determinants/thecommission/finalreport/es.

14. Behforouz HL, Drain PK, Rhatigan JJ. Rethinking the Social History. N Engl J Med. 2014;371:1277-9.

15. Vargas-Lorenzo I, Vázquez-Navarrete ML, MogollónPérez AS. Acceso a la atención en salud en Colombia. Rev Salud Pública. 2010;12:701-12.
16. Hagey J, Rulisa S, Pérez-Escamilla R. Barriers and solutions for timely initiation of antenatal care in Kigali, Rwanda: Health facility professionals' perspective. Midwifery. 2014;30:96-102.

17. Vargas J, Molina G. Acceso a los servicios de salud en seis ciudades de Colombia: limitaciones y consecuencias. Rev Fac Nac Salud Pública. 2009;27:121-30.

18. Molina G, Vargas J, Muñoz IF, Acosta JJ, Sarasti D, Higuita Y, et al. Dilemas en las decisiones en la atención en salud. Ética, derechos y deberes constitucionales frente a la rentabilidad financiera en el sistema de salud colombiano. Rev Gerenc Polit Salud. 2010; Sup 9(18):103-17.

19. Graner S, MogrenI, Duong LQ, Klingberg-Allvin M. Maternal health care professionals' perspectives on the provision and use of antenatal and delivery care: a qualitative descriptive study in rural Vietnam. BMC Public Health 2010 [visitado 2014 Sept 10]; 10:608. Disponible en: http://www.biomedcentral.com/14712458/10/608.

20. Castro-Franco BE, Peñaranda-Correa F. La comprensión de los significados de maternidad: el caso de un programa de cuidado prenatal en un centro de salud de Popayán, Colombia. Salud Colectiva. 2011;7:333-45.

21. Peñaranda F, Bastidas M, Escobar G, Torres N, Arango A. Educación para la salud: una mirada alternativa al modelo biomédico. Experiencias basadas en la evaluación del programa de crecimiento y desarrollo. Medellín: La Carreta Editores y Universidad de Antioquia; 2010.

22. Bastidas M, Torres JN, Arango A, Escobar G, Peñaranda F. La comprensión de los significados que del programa de crecimiento y desarrollo tienen sus actores: un paso hacia su cualificación. Ciencia y Saúde Colectiva. 2009;14:1919-28. 